\title{
Modélisation déterministe de la crue extrême d'un bassin versant de montagne avec application de la description géomorphologique du réseau hydrographique eterministic extreme flood modelling of a mountainous catchment with use of a geomorphologic description of the channel network
}

\author{
V. Laglaine, D. Berod, D. Devred et A. Musy
}

Volume 7, numéro 3, 1994

URI : https://id.erudit.org/iderudit/705202ar

DOI : https://doi.org/10.7202/705202ar

Aller au sommaire du numéro

\section{Éditeur(s)}

Université du Québec - INRS-Eau, Terre et Environnement (INRS-ETE)

\section{ISSN}

0992-7158 (imprimé)

1718-8598 (numérique)

\section{Découvrir la revue}

Citer cet article

Laglaine, V., Berod, D., Devred, D. \& Musy, A. (1994). Modélisation déterministe de la crue extrême d'un bassin versant de montagne avec application de la description géomorphologique du réseau hydrographique. Revue des sciences de l'eau / Journal of Water Science, 7(3), 285-308.

https://doi.org/10.7202/705202ar

\section{Résumé de l'article}

Les méthodes de calcul des crues de projet pour des grands barrages sont de type statistique ou de type déterministe (PMP/PMF). Ce dernier type de méthodes est principalement utilisé dans le monde anglo-saxon (États-Unis, Australie), mais peut offrir une alternative intéressante aux méthodes statistiques, qui présentent des lacunes pouvant être importantes.

Une application des méthodes PMP/PMF sur des bassins suisses a mis en évidence l'intérêt de ces méthodes, mais aussi leurs faiblesses. Une adaptation des outils déterministes est nécessaire pour prendre en compte les particularités des bassins de montagne. Il s'agit principalement de mieux tenir compte des propriétés physiques des bassins versants et des phénomènes de non linéarité

Dans ce but, un concept de modélisation utilisant la description géomorphologique du bassin versant a été utilisé et amélioré.

Le premier modèle utilisé est l'hydrogramme unitaire géomorphologique, qui existe depuis 1979. Le deuxième modèle utilise le concept de cascade de réservoirs hydrologiques à vidange non linéaire, dont les éléments sont issus de la description géomorphologique du bassin. C'est là une modélisation nouvelle qui est particulièrement adaptée au contexte des crues extrêmes dans les Alpes. Un travail de calibration et validation du modèle a montré que ce modèle a un bon potentiel d'utilisation. La principale difficulté revient à identifier la signification physique des paramètres du modèle, qui pourrait s'appuyer sur les résultats d'un autre type de modélisation couplant la géomorphologie du bassin aux equations de l'onde cinématique. Ce modèle est en cours de test à l'IATE. 


\title{
Modélisation déterministe de la crue extrême d'un bassin versant de montagne avec application de la description géomorphologique du réseau hydrographique
}

\author{
Deterministic extreme flood modelling of a mountainous \\ catchment with use of a geomorphologic description \\ of the channel network
}

\section{LAGLAINE, D. BEROD*, D. DEVRED, A. MUSY1}

Reçu le 23 octobre 1992, accepté le 15 octobre 1993* *.

\section{SUMMARY}

Design floods for large dams are computed either with statistical or deterministic methods (Probable maximum precipitation, PMP/probable maximum flood, PMF). The latter presents an interesting alternative to statistical methods, whose drawbacks may be significant, especially when hydrometeorological data are deficient.

PMP/PMF methods are based on assumption of the existence of an upper limit of the hydrometeorological processes. The prevalent method for PMP estimation is the maximization - transposition technique. Simpler methods, such as the statistically based technique, also exist. However, the most accurate method may well be the use of a meteorological model, but this aspect is still in the research domain.

PMF is derived from a PMP using a simplified loss function and a transfer function for which parameters are maximized. The prevalent transfer function is the unit hydrograph model.

Application of traditional PMP/PMF method on alpine catchments showed important weaknesses due to the transfer function. Alpine catchments are characterized by steep slopes, thin soil cover, poor vegetation, and large floods are due to heavy, short thunderstorms during summer. Consequently, hydrological response is very sensitive to the topography of the catchment. Nonlinearity must be taken into account in many cases. However, experience showed that a model cannot take into account a detailed description of the catchment.

In order to take into account the characteristics of alpine catchments while staying as simple as possible, we have used the geomorphologic description of the catchment as a modeling basis. This representation is based on the Strahler

1. Ecole polytechnique fédérale de Lausanne, Institut d'Aménagment des Terres et des Eaux, Hydrologie et Aménagements GR - Ecublens, 1015 Lausanne, Suisse.

* Auteur correspondant.

** Les commentaires seront reçus jusqu'au 31 mars 1995. 
ordering scheme, and defines all possible path types that a surface runoff droplet may follow to reach the outlet. This description has been used since 1979 in the geomorphologic unit hydrograph (GUH) formulation.

We developed a geomorphologic nonlinear cascade (GNC) in order to take into account nonlinear processes. The GNC model uses the geomorphologic description of the catchment to define the reservoirs of hydrological cascades. A cascade represents a path type; the first reservoir of a cascade is an overland element, and subsequent reservoirs are channels with increasing Strahler's order. Outflow from a reservoir is the inflow of the downstream reservoir. Outflows from all cascades are combined to produce the global catchment response. The two equations used in the GNC model are a global continuity equation: $I-O=d V / d t$, and a nonlinear oufflow equation: $O=k V x$, where $I$ is an input term, that is precipitation for the first reservoir, and outflow from the upstream element for channel reservoirs, $V$ is the volume of water stored in a reservoir at time $t, k$ is a constant, and $x$ an exponent. The parameter $k$ varies with each reservoir as a function of surface area (for overfand elements) or length (for channel elements). The exponent $x$ should be between 1 and 2 .

The two models, GUH and GNC, have been calibrated with an automatic optimization procedure, and tested on the Vogelbach catchment. This catchment is located in the Swiss Alps (Alptal, canton of Schwytz); it is a third order catchment, and its area is $1.55 \mathrm{~km}^{2}$. Both models gave good results, although the GUH model had a tendency to smooth the discharge.

In order to improve the physical meaning of the model parameters, we are testing a third model linking the geomorphologic description with the kinematic wave equations. Results are promising, but are not shown in this paper.

The hydrologic modeling based on the geomorphologic description of the catchment seems to be a good compromise between lumped models and detailed distributed models, which are difficult to apply.

Key-words : extreme flood, mountainous catchment, PMP/PMF, deterministic model, geomorphologic description of chatchment.

\section{RÉSUMÉ}

Les méthodes de calcul des crues de projet pour des grands barrages sont de type statistique ou de type déterministe (PMP/PMF). Ce dernier type de méthodes est principalement utilisé dans le monde anglo-saxon (Etats-Unis, Australie), mais peut offrir une alternative intéressante aux méthodes statistiques, qui présentent des lacunes pouvant être importantes.

Une application des méthodes PMP/PMF sur des bassins suisses a mis en évidence l'intérêt de ces méthodes, mais aussi leurs faiblesses. Une adaptation des outils déterministes est nécessaire pour prendre en compte les particularités des bassins de montagne. II s'agit principalement de mieux tenir compte des propriétés physiques des bassins versants et des phénomènes de non linéarité.

Dans ce but, un concept de modélisation utilisant la description géomorphologique du bassin vèrsant a été utilisé et amélioré.

Le premier modèle utilisé est l'hydrogramme unitaire géomorphologique, qui existe depuis 1979. Le deuxième modèle utilise le concept de cascade de réservoirs hydrologiques à vidange non linéaire, dont les éléments sont issus de la description géomorphologique du bassin. C'est là une modélisation nouvelle, qui est particulièrement adaptée au contexte des crues extrêmes dans les Alpes. Un travail de calibration et validation du modèle a montré que ce modèle a un bon 
potentiel d'utilisation. La principale difficulté revient à identifier la signifícation physique des paramètres du modèle, qui pourrait s'appuyer sur les résultats d'un autre type de modélisation, couplant la géomorphologie du bassin aux équations ce l'onde cinématique. Ce modèle est en cours de test à l'IATE.

Mots clés : Crue extrême, bassin versant de montagne, méthodes PMP/PMF, madèles déterministes, description géomorphologique du bassin versant.

\section{1 - INTRODUCTION}

Le dimensionnement des évacuateurs de crues des ouvrages hydrauliques majeurs teis que les grands barrages est basé sur le calcul de la crue de projet. Les méthodes statistiques classiques, telles que les ajustements de lois statistiques pour le calcul des crues de faibles fréquences (période de retour de 1000 à 10000 ans, (KITE, 1988) et la méthode du gradex (GUILLOT et DUBAND, 1967), sont largement utilisées en Europe, notamment en France et en Suisse. La majorité des pays anglophones s'intéresse à la détermination de la crue maximale probable par des méthodes déterministes de type PMP/PMF (Probable Maximum Precipitation/Probable Maximum Flood). Cette dernière méthode a fait l'objet d'une application sur des bassins versants alpins suisses, pour comparaison avec les méthodes statistiques présentant des limites d'adéquation dans le domaine des événements extrêmes. Cette étude dont les principaux résultats se trouvent dans BEROD et al. (1992) et sont rappelés dans CHAIX (1993) a pu cependant mettre en lumière certaines faiblesses de la méthode PMP/PMF pour l'application à de petits bassins versants alpins. Un projet de recherche en cours à l'Institut d'hydraulique et d'énergie (IHE) et l'Institut d'aménagement des terres et des eaux (IATE) de l'Ecole polytechnique fédérale de Lausanne (EPFL) s'attache à apporter une réflexion et une amélioration des méthodes d'estimation des crues extrêmes, par le développement d'outils déterministes adaptés au contexte suisse.

Cet article présente le concept de modélisation retenu dans le cadre de ce projet et ses possibifités d'application. La deuxième partie de l'article montre une application des méthodes PMP/PMF sur des bassins versants suisses et tente une comparaison avec les résultats des méthodes statistiques. La troisième partie propose une modélisation déterministe associée à une description géomorphologique du bassin versant. La partie suivante décrit une application des modèles développés, sur des sites suisses. Enfin, les résultats présentés permettront de tirer certaines conclusions et d'annoncer les perspectives de cette recherche. 


\section{2 - APPLICATION DES MÉTHODES PMPIPMF EN SUISSE ET COMPARAISON AVEC LES MÉTHODES STATISTIQUES}

Les méthodes PMP/PMF supposent l'existence d'une limite physique à la quantité de précipitation susceptible de tomber sur un bassin versant donné. Sous cette hypothèse, la crue résultante admet également une limite supérieure. Dans ce sens, les méthodes PMP/PMF s'opposent au concept de lois statistiques non bornées utilisées pour la détermination des crues de temps de retour de 10000 ans et plus. Datant du milieu des années trente, le concept PMP/PMF voit une constante évolution (OMM, 1973).

Une définition de la PMP est donnée par HANSEN et al. (1982) :

Théoriquement, la plus grande hauteur de précipitation d'une durée donnée, qui est physiquement possible sur une surface d'averse de taille donnée, dans un lieu géographique particulier, à une certaine période de l'année.

Une définition de la PMF est proposée par BUREAU OF RECLAMATION (1987) :

L'hydrogramme de crue d'une PMF représente les conditions d'écoulement maximales résultant de la plus sévère combinaison de conditions hydrologiques et météorologiques considérée comme raisonnablement possible pour le bassin versant étudié.

L'applicabilité des méthodes PMP/PMF a été testée sur deux bassins versants alpins suisses : la Dischma (Grisons) et la Carassina (Tessin). Leur situation géographique (bassins montagneux respectivement au nord et au sud des Alpes), leur petite taille (respectivement $43 \mathrm{~km}^{2}$ et $16,5 \mathrm{~km}^{2}$ ), et leurs caractéristiques (bassin rocheux pentu, mince couverture de sol sur un substratum gneissique à perméabilité faible, présence de zones d'éboulis pour la Dischma, proportion infime de glaciers) sont comparables à celles d'un grand nombre de bassins d'accumulation en Suisse. Le bassin de Carassina, fermé par un barrage, a été l'objet d'un redimensionnement de l'évacuateur de crue (SA UFFICIO DI INGEGNERIA MAGGIA, 1986). La crue de projet retenue pour ce redimensionnement a un temps de retour de 1000 ans, et a été estimée par des méthodes statistiques et empiriques.

- L'estimation de la PMF nécessite en premier lieu la détermination des conditions hydrométéorologiques extrêmes des bassins versants par l'analyse des événements majeurs observés sur le site, et la détermination de la hauteur maximale des précipitations sur une durée et une surface données.

Dans cette étude, différentes techniques ont été utilisées pour estimer le volume de la PMP pris comme volume de la pluie de projet. II faut noter que globalement, aucune méthode n'est très robuste. Toutefois, il est bien clair que nous ne cherchons pas à déterminer une valeur précise de la PMP, relevant de la simulation de processus atmosphériques complexes. Un ordre de grandeur vraisemblable de la PMP nous paraît suffisant pour discuter plus spécifiquement des méthodes de transfert avec leurs limites et leurs améliorations possibles. 
Les méthodes appliquées aux bassins de la Dischma et de la Carassina ont été celles empirique, statistiques et météorologique :

- Parmi les nombreuses méthodes empiriques, les unes basées sur l'établissement de courbes-enveloppes des précipitations extrêmes observées dans le monde pour des durées et surfaces données (INSTITUTION OF CIVIL ENGINEERS, 1978), les autres établies sur la base d'observations régionales intégrant des éléments météorologiques mesurés (WIESNER, 1970) ou encore représentant les PMP sous forme de courbes hauteur - surface - durée aux Etats-Unis, la méthode utilisée a été celle conduisant à la détermination de courbes dites de croissanoe à-partir d'analyses pluviométriques régionales réalisées en Grande Bretagne. Cette méthode empirique permet d'approcher la valeur maximale atteinte par une précipitation dans une région, en se basant uniquement sur les valeurs de temps de retour 5 ans (INSTITUTE OF HYDROLOGY, 1975). Les PMP empiriques de durées 6 heures et 24 heures ont pu être estimées aux stations pluviométriques relatives aux deux bassins d'étude, utilisant les valeurs de temps de retour 5 ans issues des courbes IDF.

- Une méthode dérivée des statistiques (HERSHFIELD, 1961, 1965) permet de déterminer une valeur de PMP en fonction de la moyenne $\bar{x}$ et de l'écarttype s d'une série de précipitations maximales annuelles observées :

$$
P M P=\bar{x}+K_{m} s
$$

Le facteur de fréquence $K_{m}$, égal à 15 , a été obtenu expérimentalement par l'étude d'un grand nombre de bassins des Etats-Unis. HERSHFIELD (1977) propose en outre des relations empiriques permettant de calculer $\mathrm{Km}$ er fonction de la durée de l'averse et de la moyenne de la série annuelle des pluies maximales. La méthode de Hershfield a été utilisée sur trois stations pluviographiques proches du bassin de Carassina et sur une station du bassin de la Dischma sur les périodes 1981-1990. L'application des relations empiriques de $\mathrm{Km}$ ont validé le choix de 15, pour différentes durées aux stations pluviométriques liées aux bassins. Cette méthode a permis, à partir du recueil de valeurs de précipitations maximales annuelles sur divers pas de temps, d'estimer les valeurs de PMP sur différentes durées $(3,6,12$ et 24 heures).

- Pour la détermination de la PMP, la modélisation météorologique d'averses, dont les paramètres sont maximisés dans des limites raisonnables au sens météorologique du terme (HAIDEN et al., 1990) et prenant à la fois en compte l'orographie, est complexe et loin d'être opérationnelle, par manque de données notamment à petite échelle spatiale et temporelle.

On lui a donc préféré une méthode plus simple, très répandue dans le monde, la méthode dite de maximisation-transposition. Elle nécessite toutefois des connaissances météorologiques faisant défaut à l'ingénieur hydrologue. C'est pourquoi cette étape implique la concertation des météorologues qui, par leurs compétences et leurs expériences en matière de modélisation météorologique, peuvent fournir une valeur de PMP vraisemblable et raisonnable, utilisée en entrée des modèles hydrologiques. La première étape de la technique se résume généralement en une maximisation de l'humidité seule, qui consiste à déterminer la quantité d'eau précipitable mise en jeu lors des événements majeurs observés sur le site, et à calculer la quantité maximale 
qui aurait pu précipiter dans les conditions météorologiques les plus défavorables. L'application de la maximisation nécessite alors des mesures du point de rosée et des observations de précipitations extrêmes apparaissant au cours d'une même saison. La transposition associée à la maximisation est très difficile à réaliser en milieu montagneux où l'orographie complique singulièrement la détermination des limites de transposition définissant une zone météorologiquement homogène (BEROD et al., 1992).

Pour le bassin de la Dischma, la PMP de durée 12 heures a pu être estimée par la méthode météorologique de maximisation, par le biais des travaux de l'Institut de Góographie de l'Ecole polytechnique fédérale de Zurich, en la personne de M. Grebner. Pour le bassin de Carassina situé au sud des Alpes, les phénomènes météorologiques sont beaucoup plus complexes et seule une PMP de durée 24 heures a pu être évaluée, faute de données supplémentaires en quantités et qualités suffisantes notamment pour les pas de temps inférieurs à 24 heures (seulement 9 ans de mesures pluviographiques à petits pas de temps).

Les résultats des PMP obtenues à l'aide des diverses méthodes mentionnées, pour des durées variables, sur les deux bassins d'étude, sont récapitulés dans le tableau 1. Pour simple comparaison des écarts numériques, les valeurs de précipitations de temps de retour 10000 ans, issues de la statistique des extrêmes par la construction des courbes Intensité-Durée-Fréquence aux stations pluviométriques des bassins, ont été inscrites.

Tableau 1 Estimations par différentes méthodes de la PMP et des précipitations de temps de retour 10000 ans, pour les bassins de la Dischma et de la Carassina, et pour des durées variables (échantillon de mesures pluviométriques journalières pour la période 1900-1989 pour la Dischma et 1908-1989 pour la Carassina, et à petits pas de temps pour la période 1981-1989 pour les deux bassins).

Table 1 PMP estimations with various methods, and comparisons with 10000 years return period precipitation, for the Dischma and Carassina catchments, for several rainfall durations (Daily precipitation values from 1990 to 1989 for the Dischma catchment, and from 1908 to 1989 for the Carassina catchment; small time step precipitation from 1981 to 1989 for both catchments).

\begin{tabular}{|c|c|c|c|c|c|c|c|}
\hline \multirow[b]{2}{*}{ Précipitations [mm] } & \multicolumn{4}{|c|}{ BV Carassina } & \multicolumn{3}{|c|}{ BV Dischma } \\
\hline & $3 h\left(t c^{*}\right)$ & $6 \mathrm{~h}$ & $12 \mathrm{~h}$ & $24 \mathrm{~h}$ & $6 h$ & $12 \mathrm{~h}\left(\mathrm{tc}^{*}\right)$ & $24 h$ \\
\hline PMP & & & & & & & \\
\hline - méthode météorologique & - & - & - & 370 & - & 160 & 200 \\
\hline - méthode de Hershfield & 150 & 200 & 300 & 430 & 140 & 210 & 250 \\
\hline - méthode empirique & - & 280 & - & 360 & 250 & - & 290 \\
\hline $\begin{array}{l}\text { Analyse statistique, } \\
T=10000 \text { ans }\end{array}$ & 70 & 110 & 190 & 280 & 90 & 120 & 140 \\
\hline
\end{tabular}

- Ces durées correspondent aux temps de concentration tc respectís des bassins versants. 
Notons que la méthode empirique utilisée permet de tenir compte de l'absence de valeurs importantes comme c'est le cas à la station pluviométrique de la Dischma. Cette faible pluviométrie sur la Dischma explique que les valeurs de PMP 24 heures calculées par Hershfield ou par la méthode météorologique (à partir de mesures) sont plus faibles que celles déterminées empiriquement (courbes enveloppes de valeurs mondiales ou de Grande Bretagne).

La PMP retenue pour la suite de l'étude a été celle obtenue par la méthode de Hershfield, pour une durée correspondant au temps de concentration respectif des bassins versants $(3$ heures pour la Carassina et 12 heures pour la Dischma). La méthode de Hershfield, bien que non déterministe, a été préférée aux autres méthodes, de par sa simplicité de mise en œuvre et son utilisation possible par des non-météorologues. En outre, seule cette méthode permet d'estimer des PMP pour toutes les durées inférieures à 24 heures; ce qui est intéressant pour nos bassins versants dont les temps de concentration ne sont que de quelques heures. Il est important de relever que les résultats de PMP statistique (Hershfield) trouvés sont du même ordre de grandeur que la PMP météorologique. En outre, le fait de ne disposer que de valeurs ponctuelles de PMP ne constitue pas un handicap majeur. Au vu de la taille réduite de nos bassins versants d'étude, la variabilité spatiale des précipitations peut être négligée.

- La structuration temporelle de la PMP a été assurée par différentes méthodes basées sur des hyétogrammes synthétiques construits à partir de courbes intensité-durée (Structure Chicago (KEIFER et CHU, 1957)) ou sur des structures de précipitations observées (structure selon CORDERY et al. (1984) ou structures paramétrisées (STANCULESCU et al, 1991). La méthode Chicago, moins réaliste, est à déconseiller. Basée sur les courbes IDF, la méthode Chicago suppose en effet la concomitance - peu probable - des intensités critiques sur tous les pas de temps.

- Dans l'optique d'une maximisation des paramètres de génération de la crue, les pertes sont souvent négligées sous l'hypothèse de saturation totale du sol avant l'averse (BEROD et al., 1992). Cependant, cette hypothèse doit être véri fiée pour chaque étude, car elle n'est pas plausible dans tous les cas.

Dans notre étude, les pertes ont été calculées par la méthode de production du SCS-CN (SCS,1972). L'analyse des événements pluie/débit mesurés et le contexte de l'étude nous ont encouragés à adopter des pertes initiales la quasi nulles et une valeur critique du paramètre CN égale à 90 .

- Pour la détermination de la PMF, une méthode simple de type hydrogramme unitaire est fréquemment utilisée (OMM, 1973, CHANDRA et al., 1986), au moyen des modèles OTTHYMO (JORDAN et WISNER, 1987) et HEC-1 (US ARMY CORPS OF ENGINEERS, 1990). Une modélisation non linéaire fait cependant des adeptes depuis peu, notamment en Australie, par l'intermédiaire du modèle RORB (LAURENSON et MAIN, 1992).

Dans notre étude, la fonction de transfert utilisée, de type hydrogramme unitaire de Nash, a été mise en œuvre par le truchement du modèle hydrologique OTTHYMO. La fonction de transfert de Nash est un modèle à réservoirs qui admet deux paramètres, le nombre de réservoirs $n$ pouvant ne pas être 
entier, et le temps de pointe tp de l'hydrogramme unitaire. Le calage des paramètres de Nash réalisé à partir d'événements maximaux observés sur le bassin de la Dischma, cependant de relativement faibles intensités a permis d'estimer les valeurs de $n=1,7$ et $t p=1,5$ heures. Pour le bassin de Carassina, le calage, qui a fourni les valeurs de $n=9$ et $t p=2,5$ heures, ne repose que sur un seul événement. II s'avère de plus que cette crue est la plus forte observée sur le bassin, et que son ordre de grandeur la situe bien audessus des autres événements. Les valeurs des paramètres conceptuels de l'hydrogramme unitaire proviennent d'un calage et n'ont aucune signification physique. Les valeurs de tp sont également liées aux valeurs de $n$ et peuvent expliquer les différences 8 bteñues entre les deux bassins versants. Etant donnés les problèmes de calage rencontrés sur le bassin de la Carassina, la PMF a été estimée à l'aide d'un hydrogramme unitaire synthétique plus simple développé par BUREAU OF RECLAMATION (1987). Le seul paramètre de ce modèle est le lag correspondant au temps de réponse du bassin, estimé à 150 minutes pour Carassina.

Le calage de ces modèles a soulevé le problème des mesures en milieu montagneux. Il faut en effet remarquer que le calage des fonctions de transfert s'est effectué sous une forme simplifiée, en raison du nombre restreint d'événements à disposition, voire de la mauvaise qualité des données notamment sur le bassin de Carassina. Une incertitude sur le calage peut entraîner d'importantes erreurs dans la simulation dans le domaine des crues extrêmes. En outre, le test du calage n'a pas pu être réalisé faute de données supplémentaires. Les résultats des simulations de PMF devront être alors considérés avec prudence, ayant à l'esprit les difficultés rencontrées lors du calage des fonctions de transfert et ne disposant pas d'un échantillon de validation.

Les figures 1 et 2 illustrent les simulations de PMF obtenues sur les deux bassins versants. Les résultats de la PMF et des crues calculées par les méthodes traditionnelles sont résumés dans le tableau 2.

Tableau 2 Résultats de la PMF et des crues calculées par les méthodes statistiques et empiriques, pour les bassins de la Dischma et de la Carassina.

Table 2 Results of PMF computations compared with design floods computed with statistical and empirical methods, for the Dischma and Carassina catchments.

\begin{tabular}{|c|c|c|c|}
\hline \multicolumn{2}{|c|}{ Débits $[\mathrm{m} 3 / \mathrm{s}$ ou $\mathrm{m3} / \mathrm{s} / \mathrm{km} 2]$} & Carassina & Dischma \\
\hline \multicolumn{2}{|c|}{$\begin{array}{l}\text { PMF (calculées à partir de la PMP de durée égale au temps } \\
\text { de concentration du bassin versant) }\end{array}$} & $\begin{array}{c}250 \mathrm{~m} 3 / \mathrm{s} \\
15 \mathrm{~m} 3 / \mathrm{s} / \mathrm{km} 2 \\
\text { (pour PMP } 3 \mathrm{~h} \text { ) }\end{array}$ & $\begin{array}{c}300-350 \mathrm{m3} / \mathrm{s} \\
7-8 \mathrm{~m} 3 / \mathrm{s} / \mathrm{km} 2 \\
\text { (pour PMP } 12 \mathrm{~h} \text { ) }\end{array}$ \\
\hline \multicolumn{4}{|c|}{ Méthodes traditionnelles statistiques et empiriques } \\
\hline - Ajustement statistique des débits & $\begin{array}{l}T=1000 \text { ans } \\
T=10000 \text { ans } \\
T=10000 \text { ans }\end{array}$ & $\begin{array}{c}150 \mathrm{~m} 3 / \mathrm{s} \\
-\end{array}$ & $\begin{array}{l}20-30 \mathrm{~m} 3 / \mathrm{s} \\
25-35 \mathrm{~m} 3 / \mathrm{s}\end{array}$ \\
\hline \multicolumn{2}{|l|}{ - Méthode du Gradex } & - & $140-175 \mathrm{~m} 3 / \mathrm{s}$ \\
\hline \multicolumn{2}{|l|}{ - Formules empiriques } & - & $250-350 \mathrm{~m} 3 / \mathrm{s}$ \\
\hline
\end{tabular}




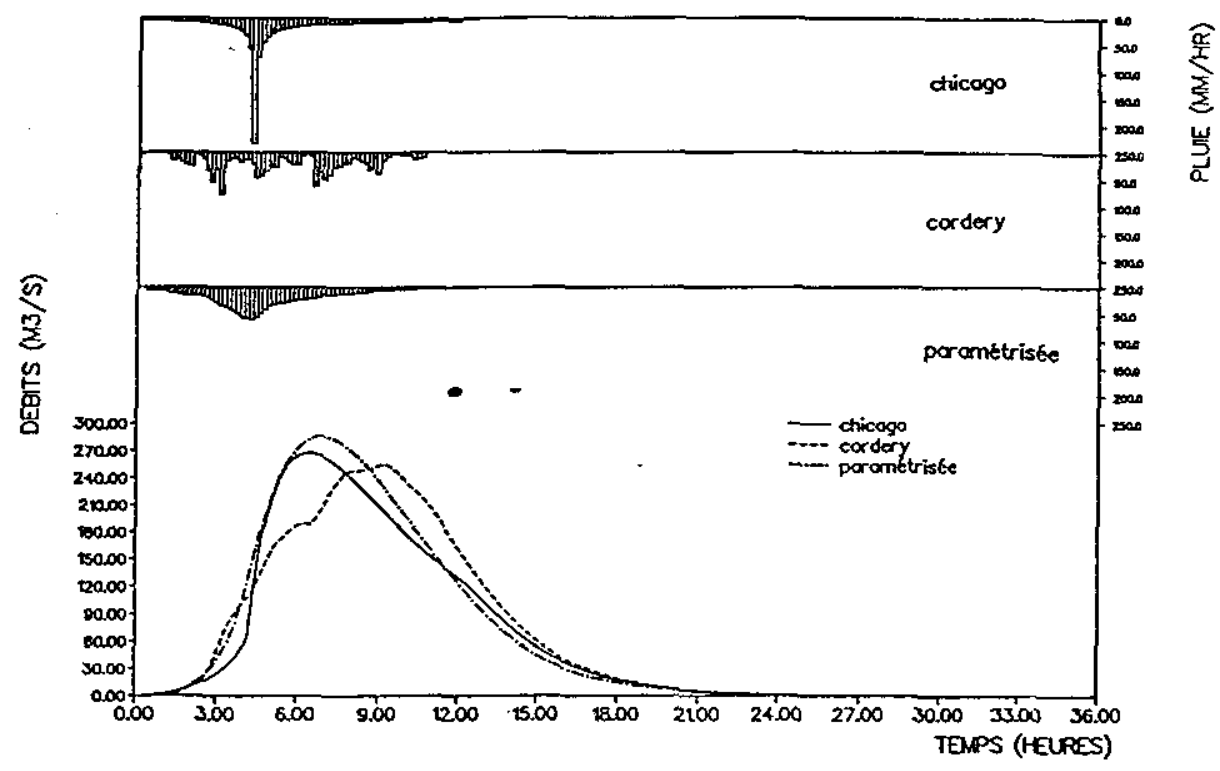

Figure 1 Estimation de la PMF à partir de différentes structures de PMP $12 \mathrm{~h}$, pour le bassin de la Dischma, avec les paramètres de calage des fonctions de production $(\mathrm{CN}=90)$ et de transfert de Nash $(n=1,7$ et $\mathrm{tp}=1,5)$.

PMF estimation on the Dischma catchment, based on a 12 hours duration PMP with various time patterns. Loss function parameter is $C N=90$, and optimized transfer function parameters are $n=1,7$ and $t p=1,5$.

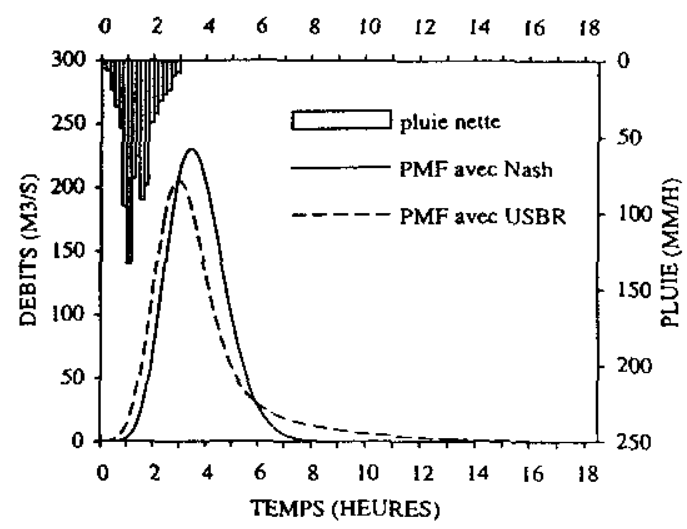

Figure 2 Estimations de la PMF pour le bassin de la Carassina en utilisant les fonctions de transfert de type hydrogramme unitaire de NASH $(n=9$ et tp $=2,5$ ) et de I'USBR (lag $=150$ minutes). La pluie de projet est une PMP de trois heures structurée selon la méthode Pilgrim et Cordery.

PMF estimation on the Carassina catchment using the unit hydrograph of Nash $(n=9, t p=2,5$ ) and the unit hydrograph of USBR (lag $=150$ minutes). Design storm is a 3 hours PMP structured according Pilgrim and Cordery's method. 
L'ajustement statistique des débits de pointe maxima annuels de la station de la Dischma de 1964 à 1989 a été réalisé sur un ensemble de lois de distribution de fréquences (Gumbel, Lognormale à 2 et 3 paramètres, Pearson lll et log-Pearson III) dont les résultats sont transcrits sous forme de bornes minimum et maximum. De même, les différentes formules empiriques utilisées, (entre autres Lauterburg, Fuller; Hofbauer; Kürsteiner ; Melli ; Coutagne, Francou et Rodier; formule rationnelle) répertoriées dans KöLLA (1986), expliquent la gamme de valeurs de débits obtenues notamment sur la Dischma. L'intérêt de recourir à diverses méthodes et formulations réside dans l'obtention d'une fourchette de valeurs de crues extrêmes permettant d'apprécier l'incertitude sur le résultat moyen.

Remarquons que l'application des méthodes statistiques n'a pas pu être réalisée correctement sur le bassin de Carassina par manque de données fiables, les débits de crue étant reconstitués à partir des enregistrements de la variation du niveau du lac de barrage de Carassina de 1968 à 1988.

Les bassins versants montrent des résultats très divergents, notamment en raison de leur situation géographique différente, la Dischma étant au centre des Alpes et la Carassina au sud. Les phénomènes météorologiques enregistrés sur le bassin de la Carassina sont beaucoup plus violents. Le calage des fonctions de transfert a également montré des comportements hydrologiques différents. Les résultats obtenus sur Carassina sont largement plus élevés que ceux de la Dischma. Notons que l'estimation de la PMF spécifique est double sur le bassin de la Carassina, pour une courte durée de PMP.

Les résultats obtenus par les méthodes PMP/PMF sont dans tous les cas supérieurs à ceux des méthodes statistiques et empiriques. Cette constatation apparaît cependant réaliste. En effet, l'extrapolation statistique se base sur des séries de données qui ne contiennent pas forcément une information sur le comportement des valeurs extrêmes. Ce phénomène est quelque peu atténué dans le cas du gradex, car le paramètre d'échelle est estimé à partir d'un échantillon important de pluies (échantillon de pluies journalières depuis 1900 à la station de Davos, pour le bassin de la Dischmal. Dans la pratique courante, la crue décamillénale est souvent comparée à la PMF. Une telle confrontation n'a pas de fondement scientifique, puisqu'elle revient à comparer deux notions d'essence différente. Les lois statistiques utilisées en hydrologie des extrêmes sont en général non bornées, alors que la PMF est censée exprimer la limite physique des phénomènes hydrométéorologiques. Les résultats obtenus ne font que conforter cette divergence.

Notons aussi que l'application des méthodes PMP/PMF en Suisse a été rendue difficile par le manque de données hydrométéorologiques de qualité suffisante. La recherche de bassins versants a montré qu'un nombre restreint était équipé de manière satisfaisante. La plupart des bassins fermés par un barrage sont dépourvus de données utiles à une étude hydrologique, statistique ou déterministe. Cependant, outre le besoin en données hydrologiques, c'est le déficit en données météorologiques qui contrarie l'application généralisée des méthodes PMP/PMF en Suisse.

La fiabilité et la précision de la détermination de la PMF sont amoindries en raison de la simplicité des méthodes utilisées. En effet, la construction d'un 
modèle météorologique fiable et adapté aux conditions alpines est encore du domaine de la recherche. En attendant, la méthode de Hershfield peut être utilisée bien qu'elle devrait être appliquée sur des séries de précipitations assez longues (au moins 20 à 25 ans contre 9 ans dans l'étude) et sachant que les résultats dépendent fortement du choix du facteur $K_{m}$, pour lequel nous ne disposons pas de valeur de contrôle mesurée. D'autre part, la réaction du bassin versant dans des conditions extrêmes ne peut être modélisée que par un outil complexe à base physique, notamment pour tenir compte des phénomènes hydrauliques en jeu lors d'une crue catastrophique. D'une manière générale, la détermination hydrologique d'une crue extrême doit être suivie d'un contrôle au niveau hydraulique, notamment pour identifier les crues débordantes. Les problèmes liés au transport solide devraient aussi être pris en considération.

Toutes ces remarques nous ont conduit à rechercher des alternatives aux méthodes simples, pour la détermination de la PMF dans les zones montagneuses.

\section{3 - PROPOSITION D'UNE MODÉLISATION APPROPRIÉE}

Le point le plus faible des méthodes PMP/PMF semble être l'hypothèse de linéarité, du moins dans le cadre d'une étude de crues extrêmes dans un contexte géographique alpin. Les crues extrêmes, provoquées par de violents orages estivaux, se manifestent principalement par du ruissellement de surface rapide dont le comportement est souvent non linéaire. De plus, la forme de l'hydrogramme unitaire, déterminée par la synthèse d'événements observés ou par un modèle conceptuel, est extrapolée abusivement et sans vérification possible au domaine des crues extrêmes. La modification supposée de la réponse hydrologique peut par contre être prise en compte par des méthodes de propagation non linéaire.

D'autre part, les bassins versants de montagne, bien que de petite taille, présentent de grandes hétérogénéités spatiales, au niveau des pentes, de la géologie, de l'occupation du sol influant sur les valeurs de perméabilité et les temps de transfert de l'eau sur le bassin. Aucune possibilité de lissage des différentes réponses dues aux hétérogénéités n'est possible pour des temps de concentration de quelques heures. Pour tenir compte de cette hétérogénéité de façon simple, une description adéquate de la géomorphologie du bassin versant peut être envisagée.

Différents modèles de simulation du ruissellement couplés à la description géomorphologique du bassin versant ont été développés et adaptés aux conditions suisses. 


\section{Description géomorphologique du bassin versant}

La description géomorphologique d'un bassin versant est réalisée dans le but de caractériser les chemins d'écoulement possibles d'une goutte d'eau tombant en un endroit quelconque du bassin. Elle s'appuie sur la schématisation du réseau hydrographique selon les ordres de Strahler (SMART, 1972). La classification de Strahler reflète la ramification du réseau hydrographique. Cette codification est basée sur les principes suivants :

- Tout cours d'eau dépourvu de tributaire est d'ordre 1.

- Le cours d'eau formé par la confluence de deux cours d'eau du même ordre iest d'ordre $i+1$.

- Le cours d'eau formé par la confluence de deux cours d'eau d'ordre différent prend l'ordre le plus élevé des deux.

- L'ordre $W$ du bassin versant est défini comme l'ordre du cours d'eau le plus élevé.

Les règles élémentaires qui régissent le comportement de l'écoulement sur un bassin selon le schéma de Strahler sont les suivantes:

- une particule de pluie nette tombe toujours sur une surface d'ordre $i$, $1 \leq i \leq W$, et non sur un cours d'eau. Cette hypothèse paraît raisonnable étant donnée la faible superficie des cours d'eau par rapport à celle des régions.

- toutes les eaux tombant sur une surface donnée d'ordre $i$ sont drainées vers un cours d'eau du même ordre $i$.

- un cours d'eau d'ordre $i$ se jette obligatoirement dans un cours d'eau d'ordre supérieur $j$, soit $i<j \leq W+1$.

- l'exutoire, d'ordre $W+1$, est l'étape finale de toutes les particules d'eau ayant cheminé sur le bassin.

Ces règles définissent l'ensemble $S$ des $2^{W-1}$ chemins possibles sur le bassin. Par exemple, les quatre chemins possibles pour un bassin d'ordre 3 sont définis selon la figure 3 .

Un chemin est caractérisé par un ensemble de $m$ éléments (région et cours d'eau) et par les changements d'état (passage d'un élément à l'autre). Le premier élément est toujours une région, les suivants sont des cours d'eau et le dernier est l'exutoire. Les éléments peuvent être représentés de plusieurs manières, soit par des réservoirs rectangulaires caractérisés par une aire (pour les régions) et une longueur (pour les cours d'eau), soit par des plans d'écoulement et des canaux définis par une longueur, une largeur, une aire. une pente et un coefficient de rugosité en vue d'une modélisation plus complexe. En outre, un chemin $s=B_{i} \rightarrow C_{i} \rightarrow C j \rightarrow \ldots \rightarrow C_{W}$ avec $R$ désignant une région et $C$ un cours d'eau, est caractérisé par la grandeur $p(s)=A_{r i} P_{c i c j}$.. $P_{c k-1 c W}$ où $A_{r i}$ est le rapport de la surface de la région d'ordre $i$ à la surface du bassin entier (la proportion de surface du bassin drainée par le chemin considéré) et $P_{c i c j}$ est la proportion de cours d'eau d'ordre i rejoignant un cours d'eau d'ordre $j$ (probabilité de transition d'un élément à un autre du chemin considéré). Cette grandeur $p(s)$ peut être assimilée à une probabilité empirique.

Un exemple numérique de calcul de probabilité des chemins est donné en référence à la description géomorphologique du bassin du Vogelbach. 


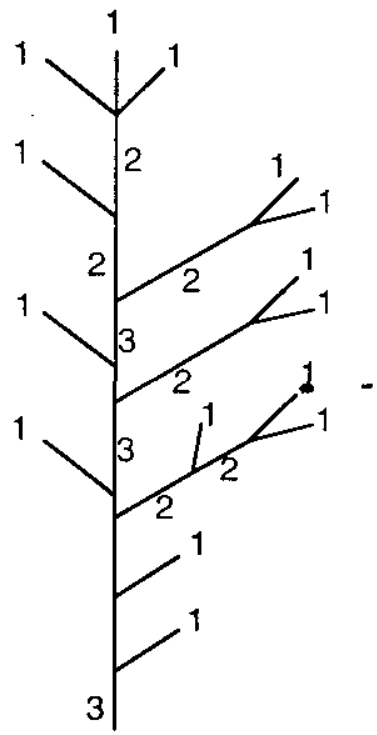

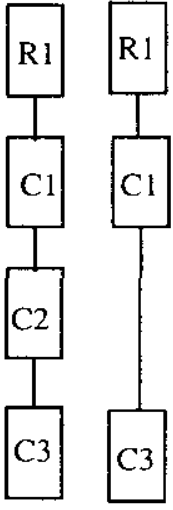

s1

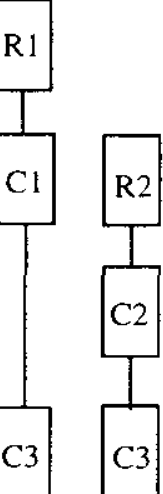

s.3

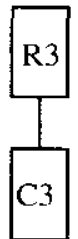

s4

Figure 3 Types de chemins possibles sur le bassin du Vogelbach d'ordre 3 (Alptal, canton de Schwytz, Suisse). $R$ représente une région, $C$ un cours d'eau. Le chiffre est l'ordre de l'élément, s est un chemin possible.

Types of possible paths for the Vogelbach catchment of order 3 (Alptal, canton of Schwytz, Switzerland). $R$ denotes a region, $C$ a channel. The figure is the element order, and $s$ is a possible path.

Parmi les caractéristiques mesurées, on a : surface de $R 1=1,033 \mathrm{~km}^{2}$, surface de R2 $=0.304 \mathrm{~km}^{2}$, surface de $R 3=0.213 \mathrm{~km}^{2}$ et la surface totale du bassin est de $1.55 \mathrm{~km}^{2}$.

$$
\begin{array}{lll}
p\left(s_{1}\right)=A_{r 1} \cdot P_{c 1 c 2} \cdot P_{c 2 c 3} & =\frac{1,033}{1,55} \cdot \frac{11}{15} \cdot \frac{4}{4}=0,4887 \\
p\left(s_{2}\right)=A_{r 1} \cdot P_{c 1 c 3} & =\frac{1,033}{1,55} \cdot \frac{4}{15}=0,1776 \\
p\left(s_{3}\right)=A_{r 2} \cdot P_{c 2 c 3} & =\frac{0,304}{1,55} \cdot \frac{4}{4}=0,1962 \\
p\left(s_{4}\right)=A_{r 3} & =\frac{0,213}{1,55} & =0,1375
\end{array}
$$

La description géomorphologique permet l'utilisation d'une gamme de modèles linéaires ou non linéaires, dont le plus simple est l'hydrogramme unitaire géomorphologique présenté ci-après.

\section{Modèle WAHS, utilisant I'hydrogramme unitaire} géomorphologique (GUH, geomorphologic unit hydrograph model)

Le modèle WAHS (Watershed Simulation Model) développé par SINGH (1989) applique la technique de l'hydrogramme unitaire géomorphologique 
(GUH) datant de la fin des années 1970 (RODRIGUEZ-ITURBE et VALDES, 1979, GUPTA et al., 1980). Le concept de base revient à assimiler l'hydrogramme unitaire $h(t)$ à la fonction de densité de probabilité $f_{B}(t)$ du temps de séjour d'une particule d'eau de pluie sur le bassin versant.

Les chemins géomorphologiques étant décrits, cette densité est obtenue par combinaison de la probabilité d'un chemin $\rho(s)$ et de la probabilité du temps de séjour sur un chemin donné $P\left(t_{s}<t\right)$, où $t_{s}$ est le temps de séjour.

La distribution du temps de séjour moyen du bassin versant, dont la dérivée est la densité recherchée, s'écrit:

$$
P\left(t_{B}<t\right)=\sum_{j=1}^{S} P\left(t_{s_{j}}<t\right) p\left(s_{j}\right)
$$

Dans cette équation (1), s est le nombre de chemins; la fonction de probabilité d'un chemin $p\left(s_{j}\right)$ peut être spécifiée entièrement par la configura tion du réseau de drainage. A l'inverse, la probabilité du temps de séjour, qui ne peut pas être dérivée directement de la géomorphologie, est modélisée arbitrairement par une loi exponentielle à un paramètre. JIN (1992) a essayé, sans amélioration significative, la loi gamma.

Finalement, la densité de probabilité du temps de séjour s'écrit :

$$
\begin{gathered}
B(t)=\sum_{j=1}^{S} \sum_{i=1}^{k} C_{i j} \exp \left(-K_{x} t\right) p\left(s_{j}\right) \\
s_{j}=<R_{j}, C_{i}, C_{j} \ldots, C_{w}>
\end{gathered}
$$

où les coefficients $C_{i m}$ sont donnés par les équations de FELLER (1971), incluant les paramètres positifs $K_{x i}$ et $m$ est le nombre d'éléments d'un chemin.

$$
c_{i m}=\frac{K_{x 1} K_{x 2} \ldots K_{x m-1}}{\left(K_{x 1}-K_{x i}\right) \ldots\left(K_{x i-1}-K_{x i}\right)\left(K_{x i+1}-K_{x i}\right) \ldots\left(K_{x m}-K_{x i}\right)}
$$

Le calcul du temps de séjour est basé sur une formule empirique de la forme $K_{b}=b A^{0,38}$, où $K_{b}$ est le temps de résidence moyen du bassin, $A$ la surface du bassin et $b$ le paramètre de calage à optimiser.

GUPTA et al. (1980) ont établi l'équivalence entre $f_{B}(t)$ et un hydrogramme unitaire $h(t)$ qui est le résultat d'une pluie nette instantanée de volume unitaire :

$$
f_{B}(t)=h(t)
$$

Le concept de base revient à définir l'hydrogramme unitaire comme une densité statistique du temps de séjour d'une particule d'eau de pluie sur le bassin versant. L'analogie est possible puisque la surface sous la courbe de l'hydrogramme unitaire vaut 1 , comme dans le cas d'une densité.

En supposant une précipitation donnée par une fonction continue, l'hydrogramme de ruissellement direct peut être construit par l'intégrale de convolution: 


$$
Q(t)=\int_{O}^{t} h(t-\tau) i(\tau) d \tau
$$

avec $Q(t)$ le débit à l'instant $t$ et $i(t)$ la pluie nette. La détermination de $Q(t)$ revient à connaître $h(t)$, et donc $f_{B}(t)$.

La description géomorphologique apporte des informations pertinentes sur l'écoulement de surface. KIRKBY (1976) s'est assuré de l'importance de la topologie du réseau hydrographique en générant des réseaux équiprobables, mais topologiquement différents. Son étude conclut que la topologie permettait d'expliquer de manière significative la crue, et que des réseaux hydrographiques différents engendraient des crues différentes.

La théorie de I'hydrogramme unitaire géomorphologique est une importante amélioration de la technique de l'hydrogramme unitaire, puisque la structure du bassin est prise en compte par sa représentation géomorphologique. Certains défauts persistent cependant, telles que les hypothèses de linéarité, pas forcément vérifiées sur les petits bassins versants alpins. En outre, le paramètre inconnu $b$, calé à partir des observations, n'est rattaché à aucune valeur physique.

Afin d'éliminer ces inconvénients tout en conservant l'avantage de la description géomorphologique, il convient d'utiliser un modèle non linéaire, dont les paramètres peuvent être déterminés par les caractéristiques physiques du bassin versant. Cet autre modèle a été développé par linstitut d'aménagement des terres et des eaux.

\section{Modèle de cascade non linéaire géomorphologique (geomorphologic nonlinear cascade -GNC- model)}

Pour tenir compte de la non-linéarité de la réponse hydrologique, un modèle à cascades de réservoirs à vidange non-linéaire est utilisé, conjointement à la description géomorphologique du bassin versant.

Le comportement de la cascade (fig. 4) est régi par deux équations :

$$
\text { équation de continuité globale : } \quad I-O=\frac{d V}{d t}
$$

équation de vidange non-linéaire: $\quad 0=k \mathrm{Vx} \quad$ si $x \neq 1$

avec / le débit d'entrée d'un élément, $O$ le débit de sortie du même élément, $V$ le stockage dans l'élément, $k$ et $x$ des paramètres caractérisant la loi de sortie de l'élément.

Le débit à l'exutoire de la cascade est calculé en résolvant le système d'équations (5), (6) pour chaque élément d'amont en aval.

La description géomorphologique permet de représenter le bassin versant par un ensemble de cascades en parallèle. Le nombre de chemins géomorphologiques déterminent le nombre de cascades, et chaque élément d'un chemin est un réservoir de la cascade. Les éléments représentant les régions 


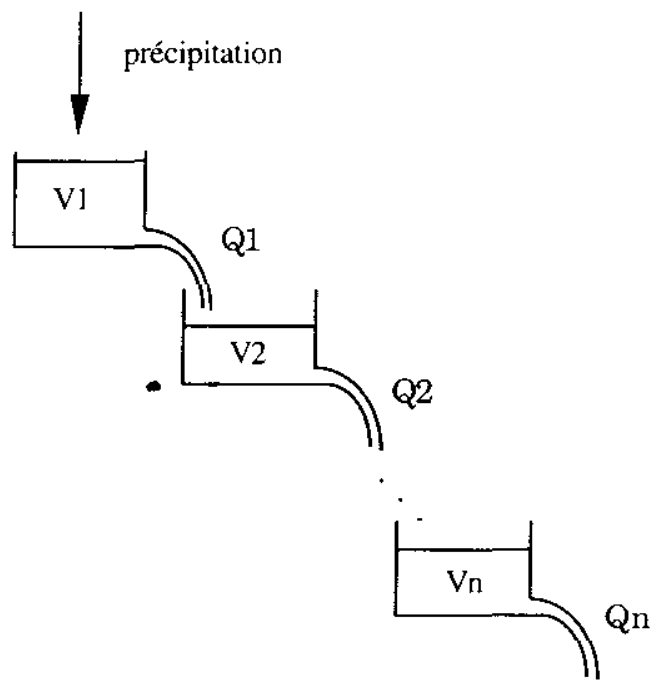

Figure 4 Exemple de cascade hydrologique.

Example of hydrologic cascade.

sont caractérisés par une surface, et les éléments désignant les cours d'eau par une longueur moyenne. L'entrée d'un réservoir « région » est la pluie, et l'entrée d'un réservoir "cours d'eau " est l'écoulement provenant du réservoir précédent. Les débits issus de chaque cascade sont ensuite combinés, en tenant compte de la probabilité du chemin $p(s)$, pour donner le débit simulé à l'exutoire du bassin versant (fig. 5).

Les deux paramètres $k$ et $x$ du modèle GNC ne peuvent pas être détermi nés directement par la géomorphologie. Ils doivent être calibrés à l'aide des observations.

L'exposant $x$, considéré comme constant pour tous les éléments, exprime le degré de non-linéarité de la réponse hydrologique.

Pour le coefficient de stockage $k$, deux formulations sont proposées dans le modèle. Dans le cas le plus simple, $k$ est considéré comme une constante $k i r$ pour tous les éléments représentant une région et une autre constante $k_{c}$ pour tous les éléments désignant un cours d'eau, quelle que soit la cascade à laquelle ils appartiennent. On parle alors de cascade non linéaire uniforme, dont les paramètres à caler sont $x_{1}$ kir et $k_{C}$

Dans l'autre cas, $k$ est exprimé comme une fonction des caractéristiques des éléments.

On a : $k i r=a A_{r}-x$ et $k_{c}=a L_{c}^{-x}$, avec $A_{r}$ la surface de la région considé rée, $L_{C}$ la longueur du cours d'eau considéré, lue sur une carte topographique, et $a$ un paramètre inconnu traduisant l'effet de pente et de rugosité des éléments. On note que $k$ et $x$ sont liés. Le calage du modèle, appelé dans ce cas cascade non linéaire non uniforme, revient à optimiser les paramètres $x$ et $a$. 


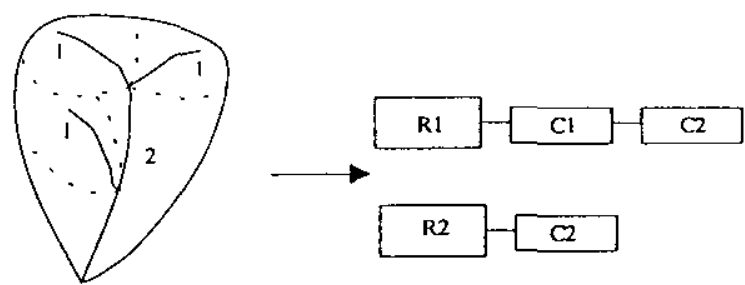

(b)

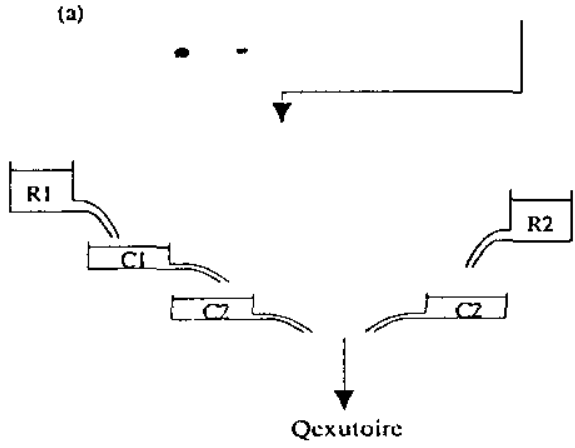

(c)

Figure 5 Exemple d'un bassin versant d'ordre 2 selon la classification de Strahler (a), description géomorphologique (b) et représentation des cascades dérivées des chemins géomorphologiques (c).

Example of an order 2 catchment classified according Strahler's method (a), geomorphologic description (b) and cascades derived from the geomorphologic paths.

Cette dernière description est plus exacte et sera retenue par la suite. Le calage est réalisé par une procédure basée sur la méthode de Rosenbrock Palmer, discutée dans ROSENBROCK (1960) et PALMER (1969). La procédure nécessite des valeurs initiales, minimales et maximales de chaque paramètre, et une fonction objective. Les valeurs initiales permettent une première simulation qui conduit à une première valeur de la fonction objective. Les paramètres sont ensuite altérés alternativement dans un sens et dans l'autre, jusqu'à ce qu'un minimum de la fonction objective soit atteint. La fonction objective retenue est :

$$
F=P \sum_{j=1}^{M}\left[Q_{p o}(j)-Q_{p c}(j)\right]^{2}+(1-P) \sum_{j=1}^{M}\left[t_{p o}(j)-t_{p c}(j)\right]^{2}
$$

où $Q_{p o}(j)$ est le débit de pointe du jème événement observé, $Q_{p c}(j)$ est le débit de pointe calculé, $t_{p o}(j)$ le temps de pointe du jème événement observé, $t_{p c}(j)$ le temps de pointe calculé, $P$ un poids compris entre 0 et 1 , et $M$ est le nombre d'événements observés. Cette fonction objective conduit au calage simultané de plusieurs événements, ce qui permet d'aboutir à une optimisation globale des paramètres. 


\section{4 - APPLICATION DES MODÈLES WAHS et GNC SUR DES SITES SUISSES}

Les modèles décrits précédemment sont encore en cours de vérification et de validation. Le calage et le test des modèles sont réalisés à partir d'événements observés et aucune valeur de PMF n'est encore avancée. L'application a pour but de montrer les divergences de résultats de simulation des deux modèles.

Le bassin versant sélecfionné pour l'essai des modèles développés est le Vogelbach, situé dans les Alpes suisses. Ses principales caractéristiques sont les suivantes (fig. 6): surface de $1,5 \mathrm{~km}^{2}$, altitude comprise entre 1050 et $1545 \mathrm{~m}$, coefficient de Gravélius égal à 1,05 (forme arrondie), pente jusqu'à $50 \%$, épaisseur de sol jusqu'à $50 \mathrm{~cm}$ et couverture de sol forestière et agricole.

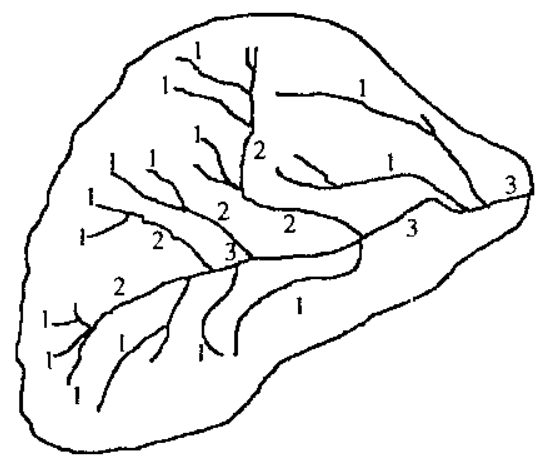

\begin{tabular}{|l|c|}
\hline Ordre du bassin & 3 \\
$\sum \mathrm{C}$ d'ordre 1 & 15 \\
$\sum \mathrm{C}$ d'ordre 2 & 4 \\
$\sum$ chernins & 4 \\
$\sum \mathrm{C}$ dans chemin 1 & 11 \\
$\sum \mathrm{C} 1$ dans chemin2 & 4 \\
$\sum \mathrm{C} 2$ dans chemin2 & 4 \\
Surface de R1 & 2.9 à $18 \mathrm{ha}$ \\
Surface de R2 & 3 à $15 \mathrm{ha}$ \\
Surface de R3 & 21 ha \\
Longueur de C1 & 150 à $1000 \mathrm{~m}$ \\
Longueur de C2 & 300 ̀े $650 \mathrm{~m}$ \\
Longueur de C3 & $1100 \mathrm{~m}$ \\
\hline
\end{tabular}

Figure 6 Carte du bassin versant du Vogelbach $\left(1,55 \mathrm{~km}^{2}\right)$, définition des ordres de Strahler (certains petits cours d'eau ont été négligés) et principales caractéristiques géomorphologiques du bassin dérivées de la carte topographique. La description géomorphologique du Vogelbach avec la représentation des chemins possibles est montrée sur la figure 3.

Map of the Vogelbach catchment $\left(1,55 \mathrm{~km}^{2}\right)$, definition of Strahler orders (minor channels are neglected), and main geomorphologic features as derived from the map. Geomorphologic description of the Vogelbach catchment and the possible paths are shown on figure 3.

Des données pluie/débit, globalement de bonne qualité, ont été mises à disposition pour le bassin depuis 1974, par l'institut fédéral de recherches sur la forêt, la neige et le paysage (FNP-Birmensdorf). Les événements de crue les plus importants apparaissent en été et sont dus à des épisodes orageux de courte durée. Au vu des caractéristiques du bassin, notamment des pentes importantes et une faible épaisseur de sol, il paraît raisonnable d'énoncer que le processus de crue dominant est le ruissellement de surface. 
Les deux modèles ont été calibrés pour le bassin versant de Vogelbach. Ils utilisent en entrée une pluie nette, déterminée à partir d'une pluie brute observée ou simulée diminuée des pertes par infiltration, elles-mêmes calculées par la fonction de Philip ou la méthode du SCS-CN. Les hydrogrammes de ruissellement direct ont été obtenus par une technique de séparation des écoulements.

A partir d'une quinzaine d'événements pluie/débit retenus, deux ensembles ont été choisis : un ensemble de 7 événements pour le calage des modèles et un ensemble des événements restants pour la validation. Les caractéristiques principales des événements retenus sont indiquées dans le tableau 3.

Tableau 3 Principales caractéristiques des événements retenus pour l'application des modèles.

Table 3 Main characteristics of the events used for models applications.

\begin{tabular}{|c|c|c|c|c|c|}
\hline \multicolumn{3}{|c|}{$\begin{array}{l}\text { Caractéristiques des averses observées } \\
\text { [MIN - MAX] }\end{array}$} & \multicolumn{3}{|c|}{$\begin{array}{c}\text { Caractérlstiques des hydrogrammes } \\
\text { rulsselés [MIN - MAX] }\end{array}$} \\
\hline $\begin{array}{l}\text { Hauteur totale } \\
\qquad(\mathrm{Mm}]\end{array}$ & $\begin{array}{l}\text { Durée totale } \\
\text { [min] }\end{array}$ & $\begin{array}{l}\text { Int. } \max \\
{[\mathrm{mm} / \mathrm{t}]}\end{array}$ & $\begin{array}{l}\text { Lame ruisselée } \\
\text { [mm] }\end{array}$ & $\begin{array}{l}\text { Qp } \\
{[1 / s]}\end{array}$ & $\begin{array}{l}\text { CR }[\%] \\
9-60\end{array}$ \\
\hline $12-88$ & $100-1430$ & $9-103$ & $1-27$ & $285-4910$ & $9-60$ \\
\hline
\end{tabular}

Pour chacun des modèles, le jeu de paramètres, obtenu par optimisation sur les événements de calage simulés, est appliqué à l'ensemble des événements de test, pour validation. La figure 7 présente le résultat du calage et du test des modèles sur deux événements différents. La validation du modèle CNG en particulier indique que le modèle est apte à simuler des crues sur le bassin versant étudié en utilisant une schématisation du réseau hydrographique bien définie.

La représentation des résultats des simulations, sous forme de comparaison entre les débits de pointe des hydrogrammes de crue observés et les débits de pointe des hyơrogrammes simulés d'une part et entre leurs temps de pointe d'autre part, permet de visualiser la qualité des simulations et de proposer un choix préférentiel de l'un ou l'autre des modèles, pour le bassin versant étudié. Ces graphes sont dessinés pour les deux modèles, avec les résultats des simulations des événements de test, dans la figure 8 . Les deux modèles reconstituent les événements de manière satisfaisante.

L'exercice de calage a permis de vérifier les limites de l'hypothèse de iinéarité du modèle WAHS sur nos bassins et que le paramètre $b$ n'a aucune signification physique. Outre un effet de lissage parfois important, les simulations ont montré que la décrue simulée par le modèle est souvent trop rapide.

Le modèle GNC s'est avéré facile d'utilisation, une fois les paramètres géomorphologiques déterminés. La principale difficulté réside dans l'estimation et la signification des paramètres du modèle. De manière générale, le modèle 


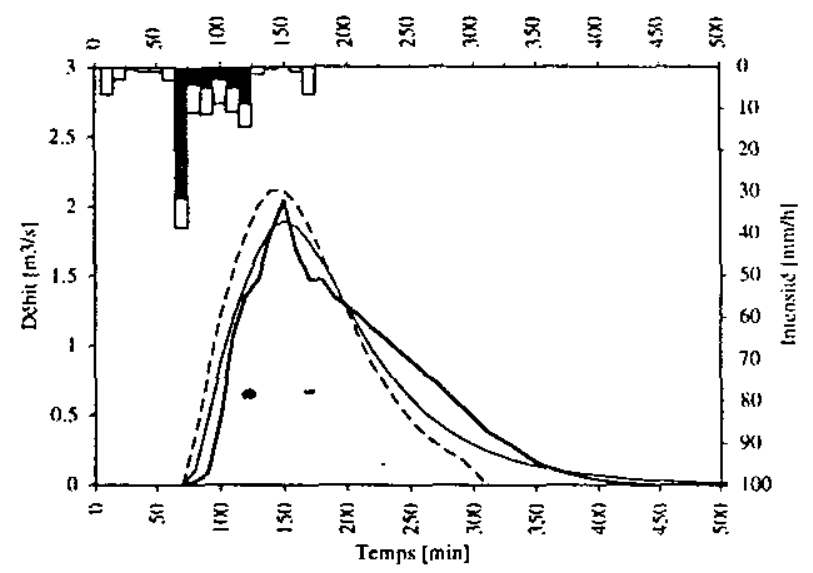

(a) Calage sur l'événement du 9 juillet 1987

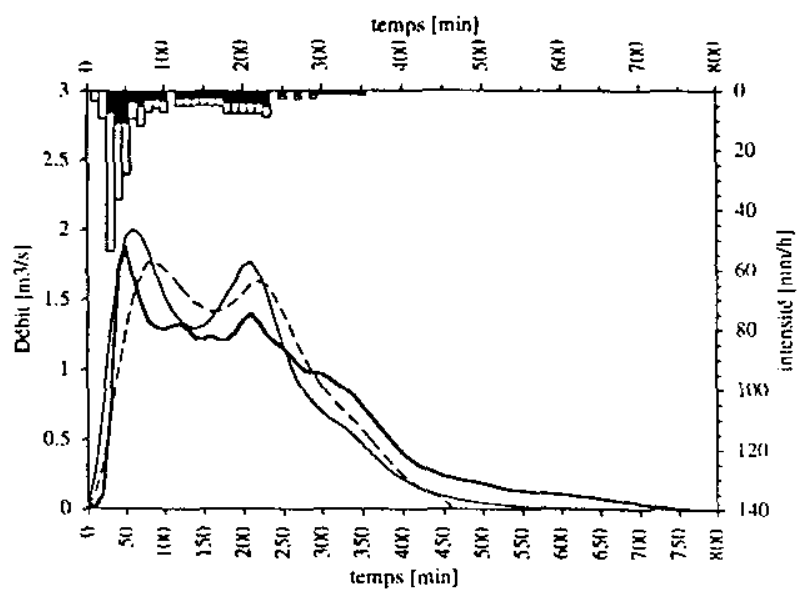

(b) Test sur l'événement du 18 juillet 1986

Figure 7 Application des modèles WAHS et GNC non uniforme, sur le Vogelbach ; (a) exemple de calibration; (b) exemple de test.

Application of the WAHS and GNC models on the Vogelbach catchment; (a) example of calibration run; (b) example of validation run.

GNC donne des résultats satisfaisants, malgré sa tendance à lisser quelque peu les hydrogrammes simulés. Ce phénomène est certainement dû à une trop grande globalisation des processus. Une voie d'amélioration possible est la recherche de relations entre les paramètres du modèle, pour le moment obtenus par optimisation lors du calage, et les caractéristiques physiques du bassin versant. 

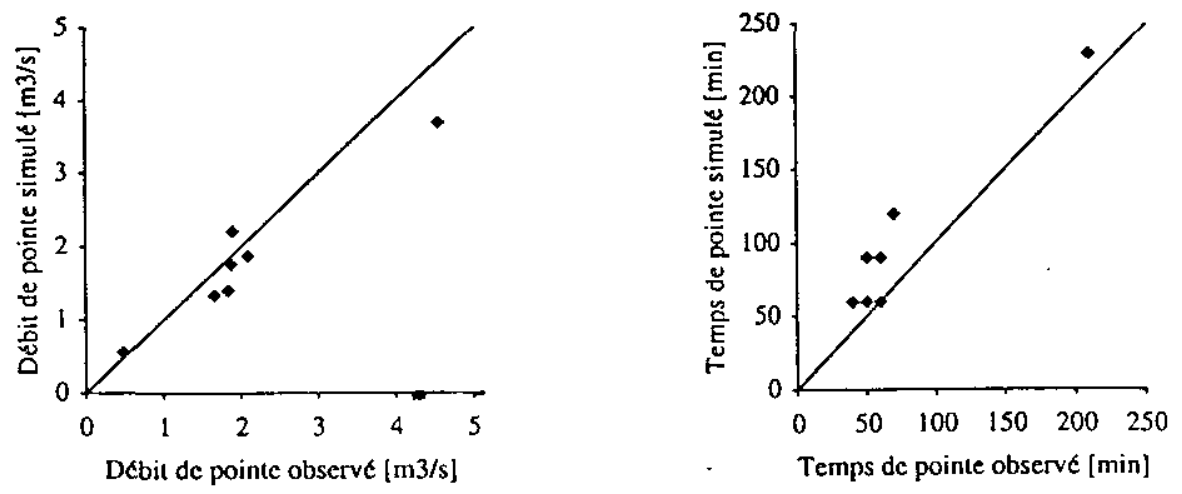

(a) Modele WAHS
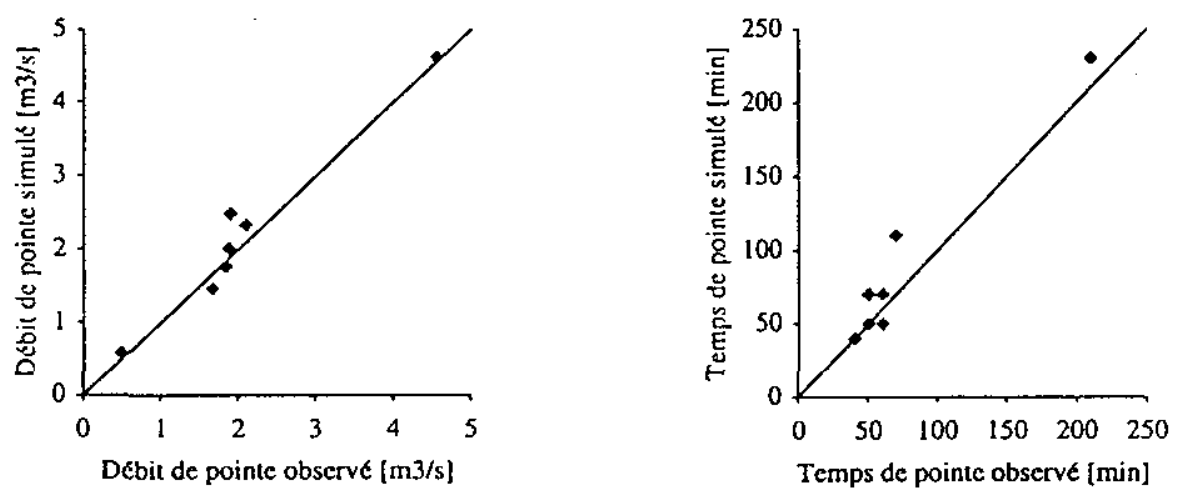

(b) modèle GNC

Figure 8 Résultats des tests des deux modèles : représentation des débits de pointe des hydrogrammes de crue observés en fonction des débits de pointe des hydrogrammes simulés, même graphe pour les temps de pointe observés et simulés. Le dessin de la ligne de partaite correspondance (perfect agreement line) facilite la comparaison.

Results of the validation of the two models. Graphics show observed vs. simulated peak flow, and observed vs. simulated time to peak for validation runs. Perfect agreement line is also represented.

Sachant que les processus en jeu lors d'événements extrêmes sur nos bassins sont non linéaires, le modèle GNC paraît de ce fait mieux adapté. De plus, les paramètres qu'il utilise ont une meilleure représentation physique que le paramètre $b$ du modèle WAHS. 


\section{5 - PERSPectives et CONClusions}

Les méthodes PMP/PMF, largement utilisées dans le monde anglo-saxon, ne peuvent être ignorées par l'ingénieur hydrologue. Elles représentent en Suisse une alternative aux méthodes statistiques habituellement utilisées pour la conception d'ouvrages hydrauliques à haute exigence de sécurité. Elles doivent être testées dans le cadre de la vérification du dimensionnement des évacuateurs de crue des grands barrages. L'avantage des méthodes PMP/ PMF vis-à-vis des méthodes traditionnelles se manifeste dans leur détachement par rapport aux hypothèses fortes des méthodes statistiques et par la prise en compte de caractéristiques physiques des phénomènes hydrométéorologiques. Cependant, outre les critiques formulées à l'égard du concept luimême, les méthodes PMP/PMF présentent des faiblesses, dans leur application en milieu alpin, liées aux hypothèses de linéarité et à la non prise en compte des caractéristiques physiques des bassins versants.

Dans l'optique d'améliorer les méthodes existantes de calcul de crues extrêmes qui s'avèrent mal adaptées au milieu alpin, deux modèles ont été proposés, avec comme atout commun la prise en compte de la description géomorphologique du bassin versant. Ce sont le modèle WAHS utilisant l'hydrogramme unitaire géomorphologique et le modèle de cascade non linéaire géomorphologique GNC. Ces modèles sont adaptés aux objectifs désirés, à savoir la modélisation de crues extrêmes sur de petits bassins versants alpins. De plus, la description géomorphologique du bassin permet de déterminer l'apport relatif des différents types de chemins simulés. Cependant, certaines difficultés persistent et des améliorations des modèles sont envisagées pour la poursuite de l'étude. En premier lieu, notons que le choix de la fonction d'infiltration revêt une grande importance dans la structure temporelle de la pluie nette pour laquelle les modèles sont très sensibles. En outre, les modèles seront utilisés selon leurs spécificités, de façon à déterminer l'importance relative des facteurs générateurs de la crue extrême: les pentes, le réseau hydrographique et la rugosité. Ce demier paramètre lié à la couverture du sol nécessitera une étude particulière pour déterminer le coefficient de frottement représentatif des phénomènes naturels et sa variation. Tous ces paramètres seront utilisés par couplage avec les équations de l'onde cinématique. A cet effet, un modèle d'onde cinématique associé à la description géomorphologique du bassin a été développé à l'IATE et demande encore à être validé. Néanmoins les résultats des simulations déjà acquis à partir de ce modèle sont largement encourageants et vont dans le sens d'une meilleure représentation physique des processus observés lors d'événements extrêmes, en zone montagneuse.

Le calage et la validation des modèles, ainsi que l'analyse de leur sensibilité à la variation des paramètres, devront être réalisés sur d'autres bassins versants alpins en vue d'une généralisation. En parallèle, la signification physique des paramètres, jusqu'à présent calés à partir des observations, leur lien avec des caractéristiques du bassin versant et leur importance relative constituera un sujet de préoccupation essentiel pour la modélisation hydrologique. On se doit de souligner ici l'intérêt de ce lien avec la physique du bassin qui permet l'utilisation des modèles sur des bassins versants non jaugés. 
Pour sortir du cadre des modèles développés par l'IATE et en amont de cette étude, des améliorations sont attendues dans différentes voies : la modélisation physique des précipitations extrêmes et notamment de la PMP, l'utilisation des SIG pour décrire les caractéristiques entre autres géomorphologiques des bassins versants et générer les paramètres d'entrée des modèles de simulation de la PMF.

\section{REMERCIEMENTS}

Les auteurs tiennent à remercier le Prof. Singh, de l'université de Louisiane, pour avoir mis à disposition le modèle WAHS, et pour son aide au cours du développement du modèle GNC. Les données utilisées proviennent de l'Institut Fédéral de recherche sur la Forêt, Neige et Paysage (FNP) à Birmensdorf, Suisse.

\section{RÉFÉRENCES BIBLIOGRAPHIQUES}

BEROD D., DEVRED D., LAGLAINE V., CHAIX O., ALTINAKAR M., DELLEY P., 1992. Calcul des crues extrêmes par des méthodes déterministes de type pluie maximale probable (PMP)/crue maximale probable (PMF). Application au cas de la Suisse. Rapport de I'Institut d'Aménagement des Terres et des Eaux, Ecole polytechnique Fédéral.e de Lausanne.

BUREAU OF RECLAMATION (1987). Design of small dams. US Bureau of Reclamation, Washington.

CHAIX O., 1993. Calcul de la crue maximale probable. Wasser Energie Lutt, 5/6 : 114118.

CHANDRA M., MUTREJA K.N., JINDAL R.K., 1986. Probable maximum flood for Lakhwar dam. International symposium on hydrological aspects of mountainous watersheds, University of Roorkee: V-24-V-31.

CORDERY I., PILGRIM D.H., ROWBOTTOM A., 1984. Time patterns of rainfall for estimating design floods on a frequency basis. Water Sci. Tech, 16: 155-165.
FELLER W., 1971. An introduction to probability theory and its applications, Vol 2. John Wiley and Sons, New York.

GUILLOT P., DUBAND D., 1967. La méthode du gradex pour le calcul de la probabilité des crues à partir des pluies. AlSH Publication, 84 : 560-569.

GUPTA V.K., Waymire E., Wang C.T., 1980. A representation of an instantaneous unit hydrograph from geomorphology. Water Resour. Res., 16 (5) : 855-862.

HAIDEN T., KAHLIG P, KEPSCHBAUM M. NOBILIS F., 1990. On the influence of mountains on large scale precipitation : a deterministic approach towards orographic PMP. Hydrol. Sci. J., 35 : 501-510.

HANSEN E.M., SCHREINER L.C., MILLER J.F., 1982. Application of probable maximum precipitation estimates - United States East of the 105th Meridian. NOAA Hydrometeorological. Report no 52, Washington.

HERSHFIELD D.M., 1961. Estimating the Probable Maximum Precipitation. J. Hydraut. Div. ASCE, 87 : 99-106. 
HERSHFIELD D.M., 1965. Method for Estimating Probable Maximum Rainfall. J. Am. Wat. Wks Ass., 57(8) : 965-972.

HERSHFIELD D.M., 1977. Some tools for hydrometeorologists. 2nd Conference on Hydrometeorology, American Meteorological Society, Boston MA.

INSTITUTION OF CIVIL ENGINEERS, 1978. Floods and reservoir safety. Institution of Civil Engineers, Londres.

INSTITUTE OF HYDROLOGY, 1975. Floods studies report, vol II: Meteorological studies. Withefriars Press Lts, London.

JIN C.X., 1992. A deterministic gamma-type geomorphologic instantaneous unit hydrograph based on path types. Water Resour. Res., 28 (2) : 479-486.

JORDAN J.P., WISNER, P., 1987. Description du modèle OTTHYMO et exemples d'application. Institut d'Aménagement des Terres et des Eaux, Ecole polytechnique Fédérale de Lausanne.

KEIFER J.C., CHU H.H., 1957. Synthetic storm patterns for drainage design. $J$. Hydraul. Div. ASCE, 83(4).

KIRKBY M.J., 1976. Test of the random network model, and its application to basin hydrology. Earth Surface Processes, I, 197-212.

KITE G.W., 1988. Frequency and risk analyses in hydrology. Water Resources Publications.

KOLLA E., 1986. Zur Abschătzung von Hochwassern in Fliessgewăssern an Stellen ohne Direktmessungen. Mitteilung der Versuchsanstalt für Wasserbau, Hydrologie und Glaziologie, ETHZ.

LAURENSON E.M., MAIN, R.G., 1992. RORB - Version 4, runoff routing program, user manual. Monash University, Dept of Civil Engineering, Australie.

MUSY A., SOUTTER M., 1991. Physique du sol. Presses Polytechniques et Universi- taires Romandes, Collection Comment gérer l'environnement, 6, Lausanne.

OMM, 1973. Manual for Estimation of Probable Maximum Precipitation. Secrétariat de l'organisation mondiale de météorologie. Genève, n 332 .

PALMER J.R., 1969. An improved procedure for orthogonalizing the search vectors in Rosenbrock's and Swann's direct search optimization methods. Comput. J., 12 : 69-71.

RODRIGUEZ-ITURBE I., VALDES J.B., 1979. The geomorphologic structure of hydrologic response. Water Resour. Res., 15 (6) : 1409-1420.

ROSENBROCK H.H, 1960. An automatic method for finding the greater or least value of a function. Comput. J., 3 (3) : 175-184

SA UFFICIO DI INGEGNERIA MAGGIA, 1986. Studio idrologico sul bacino imbrifero del rial.e Carassina. SA Ufficio di ingegneria Maggia, Locarno.

SCS, 1972. National Engineering Handbook, section 4 - hydrology. Soil Conservation Service, Dept. of Agriculture, USA.

SINGH V.P., 1989. A quasi-conceptual linear model for synthesis of direct runoff with potential. Application to ungaged basins. Military Hydrology, Report 17, US Army Corps of Engineers.

SMART J.S., 1972. Channel networks. Advan. Hydrosci., 8 : 305 - 346.

STANCULESCU M.B., BEROD D., DEVRED D., LAGLAINE V., 1991. Structuration synthétique des averses par modélisation des paramètres morphologiques. Rencontres hydrologiques Franco-Roumaines, Paris, 2-4 septembre.

US ARMY CORPS OF ENGINEERS, 1990. HEC-1 flood hydrograph package, user's manual. US Army Corps of Engineers, Hydrologic Engineering Center, Davis, USA.

WIESNER C.J., 1970. Hydrometeorology. Chapman and Hall Ltd, London. 